\title{
Fullerene in Carbon Nanoring: A Saturn-Like Supramolecular Complex
}

\author{
Jie Zhang, ${ }^{a, b}$ Chunru Wang, ${ }^{a}$ and Taishan Wang ${ }^{*, a}$ \\ ${ }^{a}$ Beijing National Laboratory for Molecular Sciences, Key Laboratory of Molecular Nanostructure and \\ Nanotechnology, Institute of Chemistry, Chinese Academy of Sciences, Beijing 100190, China \\ ${ }^{b}$ University of Chinese Academy of Sciences, Beijing 100049, China
}

Email: wangtais@iccas.ac.cn (T. W.)

\begin{abstract}
Fullerenes have attracted much attention because of their unique electronic structure and spherical cage shape. Specially, fullerenes have been widely used as guest in supramolecular chemistry due to their spherical structures. Recently, the supramolecular complexes of fullerenes and several types of carbon nanorings have been constructed and investigated. The complex of fullerene in carbon nanoring is like a Saturn, and this unique kind of supramolecular complexes will have potential applications in chemical, material and physical fields. In this review, various complexes of fullerene in carbon nanoring and their special properties are described. We hope to expand the application of these supramolecular complexes in various fields.
\end{abstract}

Keywords fullerene, carbon nanoring, host-guest interaction, supramolecular complex

\section{Introduction}

Since 1985, the fullerenes have been widely studied and many applications have been found for this cage-shaped carbon nanomaterials. ${ }^{[1]}$ Fullerenes and fullerene derivatives are well-known spherical aromatic molecules that have unique properties, such as electron affinity, which makes fullerenes attractive for photovoltaic device applications. Furthermore, fullerenes can be easily chemically modified to improve the water solubility and then to develop their application as nano medicine. ${ }^{[2]}$

Specially, fullerenes have been widely used as guest in supramolecular chemistry due to their spherical structures. As guest molecules, fullerenes have been entrapped in cyclodextrin, porphyrin, large aromatic compounds, porous materials, etc., and varied host-guest interactions have been disclosed. $^{[3]}$ In these kinds of supramolecular systems, many chemical and physical phenomenons have been revealed. Recently, the supramolecular complexes of fullerenes and several types of carbon nanorings have been constructed and investigated. For example, the cycloparaphenylenes (CPPs) and their analogs have attracted wide attention because of their beautiful nanoring structure and $\pi$-conjugated system. ${ }^{[4]}$ Nanoring of CPPs can easily form a Saturn-like supramolecular structure with fullerenes. As far as we know, the term "Nano Saturn" was originally used in the theoretical research. In 2016, McKee et al. reported their research results entitled "Saturn system", which is about a theoretical study of supramolecular complexes of various hydrocarbon nanocycles and $\mathrm{C}_{60}{ }^{[5]}$ In this minireview, various complexes of fullerene in carbon nanoring and their special properties are described.

\section{Supramolecular Complexes of Fullerene and Carbon Nanoring}

In the current literature reports, the ring moieties are divided into two categories according to their shape. The first type is the belt-like ring, whose benzene plane adheres to the guest fullerene via $\pi-\pi$ interaction. This type of nanoring is represented by the cycloparaphenylenes (CPPs). Because of the large contact area between the ring and the guest molecule, they can be closely combined. The second type is the disk-like ring. This type of ring interacts with the fullerene mainly through $\mathrm{CH}-\pi$ (or X-T) interactions. ${ }^{[6]}$ Herein, we mainly introduce the supramolecular complexes of fullerene with above two types of carbon nanoring.

In 2003, Kawase and Oda et al. reported a supramolecular complex using CPPA(cyclic paraphenyleneacetylene) as the host molecule. The complex of bis (ethoxycarbonyl) methanofullerene and [6]CPPA was crystallized and X-ray crystallographic data confirmed the orientation of the CPPA phenyl rings in proximity to the [5:6] fullerene fusions (Figure 1). ${ }^{[7]}$ Subsequently, they used other fullerenes and larger CPPA derivatives to study their associative properties. Briefly, the [6]CPPA and [7]CPPA have similar association ability to $C_{70}$, while the [8]CPPA size is larger and its association ability to fullerene is weaker. These results indicate that the cavity size is an important factor in host-guest association. ${ }^{[8]}$
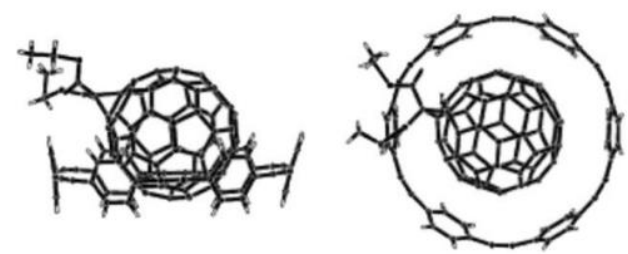

Figure 1 Crystallographic structures of a [6]CPPA and methanofullerene complex.

Although CPPA derivatives tend to form tight complexes with fullerene, their unstable nature hinders further experimental studies. On the contrary, completely $\mathrm{sp}^{2}$-hybridized CPPs(cycloparaphenylenes) derivatives are sufficiently stable and can similarly encapsulate fullerene molecules. ${ }^{[9]}$ Despite their simple structures, however, the synthesis of CPPs was only achieved in 2008 from curved molecular precursors after intensive efforts by Jasti and co-workers. ${ }^{[10]}$ Afterwards, Iwamoto et al. reported initial saturn-like complexes of CPPs with fullerene. They found that 
$\mathrm{C}_{60}$ can be selectively encapsulated by [10]CPP (Figure 2). This finding opens the possibility of utilizing CPPs as size- and shape-selective host molecules for various fullerenes. Since several CPPs with different sizes are available, they would also serve as selective hosts for certain fullerenes depending on their varied size. ${ }^{[11]}$

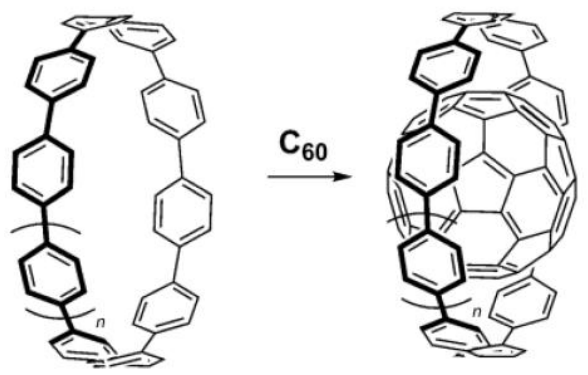

Figure 2 Schematic complexation of cycloparaphenylene (CPP) and $\mathrm{C}_{60}$.

In 2015, Yuan et al. explored the intrinsic nature of $\pi^{-} \pi$ noncovalent interactions of the CPPs and fullerene host-guest systems using computational method. The position orientation of $\mathrm{C}_{70}$ in [ $n$ ]CPP ( $n=10,11$ and 12) was studied emphatically. According to the relative values of the binding energies and thermodynamic information, the guest $\mathrm{C}_{70}$ is apt to adopt lying, standing, and half-lying orientations in the cavities of [10]CPP, [11]CPP, and [12]CPP, respectively (Figure 3). ${ }^{[12]}$ These results suggest that size and shape fitting are very important for the relative orientation of fullerene in these supramolecular complexes.

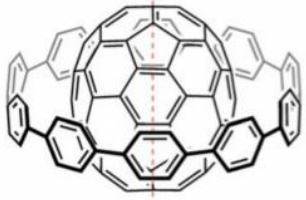

$[10] \mathrm{CPP} \supset \mathrm{C}_{70}$

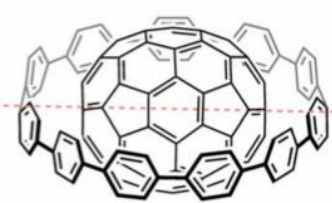

[11] $\mathrm{CPP} \supset \mathrm{C}_{70}$
Figure 3 Structures of CPP complexes with $\mathrm{C}_{70}$. Red broken lines show the long axis of ellipsoidal $\mathrm{C}_{70}$ molecule.

In 2018, Xu et al. achieved the synthesis of two [2] rotaxanes consisting of one [10]CPP moiety binding to a central fullerene with bis-adduct binding site and another two fullerene hexakisadduct stoppers using a concave-convex $\pi-\pi$ template strategy. ${ }^{[13]}$ Later, Wegner and coworkers investigated the complex between the azafullerene dimer $\left(\mathrm{C}_{59} \mathrm{~N}\right)_{2}$ and [10]CPP. Two stage binding constants were determined to be $K_{\mathrm{a} 1}=8.4 \times 10^{6} \mathrm{M}^{-1}$ and $K_{\mathrm{a} 2}=3.0 \times 10^{6} \mathrm{M}^{-1}$, respectively. ${ }^{[14]}$

In 2019, Du, Yang and co-workers achieved the synthesis of two novel $\pi$-extended crown-like molecules (TCR and HCR) with embedded curved nanographene units. They proposed a strategy to further increase the binding affinity of $[n]$ CPPs for fullerenes is to increase the $\pi$-surface by inserting annelated moieties into the ring (Figure 4). Moreover, the photocurrent experiments showed that these complexes can form photoconductive heterojunctions. It is helpful to apply the complex to the field of optoelectronic materials. ${ }^{[15]}$

In order to mimic the Saturn shape precisely, it is better to use a planar disk-shaped ring to include a spherical fullerene. lyoda et al. synthesized oligothiophene macrocycles with ethynylene or ethenylene linkers. High resolution STM investigation of monolayers revealed that the presence of two individual bithiophene units as well as the size of the macrocycle led to the formation of a superstructure with a $1: 2$ a)

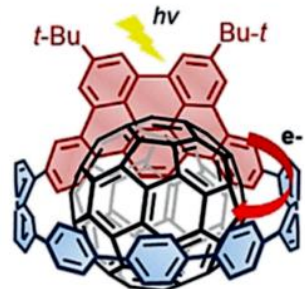

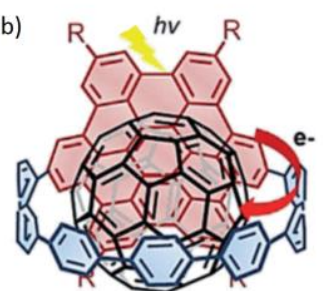

Figure 4 Schematic structures of a) $T C R \supset \mathrm{C}_{60}$ and b) $\mathrm{HCR} \supset \mathrm{C}_{60}$.
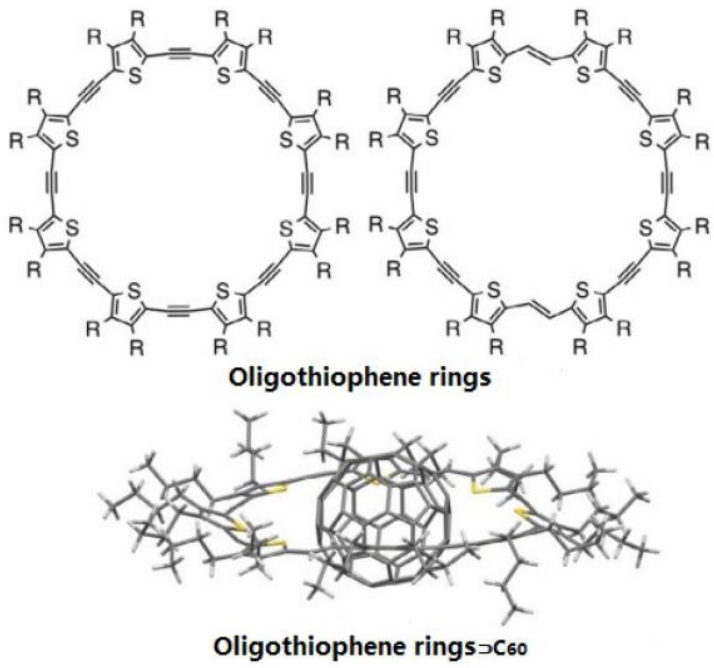

Figure 5 Structure of oligothiophene rings and crystallographic structure of oligothiophene rings $\supset \mathrm{C}_{60}$.

stoichiometry. The fullerene units were located around the periphery of the bithiophene units, indicating the donor-acceptor interaction between $\mathrm{C}_{60}$ and the electron-rich bithiophene units (Figure 5). In addition, they found that the inner cavities of oligothiophene macrocycles are easily adjustable to guest molecules through selecting numbers of 2,5-thienylene, ethynylene, and vinylene units. Thus, they concluded the cavity size of macroring can adjusted fullerenes to produce Saturn-like complexes. ${ }^{[16]}$

Okada et al. theoretically investigated the possibility of a carbon nanomaterial with Saturn shape as a novel inclusion compound consisting of $\mathrm{C}_{60}$ and cyclohexabiphenylene based on total energy calculations using density functional theory. They found that nano-Saturn is energetically stable with similar or lower total energy to that of the other $\mathrm{C}_{60}$ inclusion compounds experimentally synthesized to date. Later, they theoretically investigated a nanoscale model of Saturn consisting of $\mathrm{C}_{60}$ and [24]circulene as a novel inclusion compound (Figure 6). Because this molecule is fully conjugated and completely planar, it might be an ideal ring motif for nano-Saturns. ${ }^{[17]}$
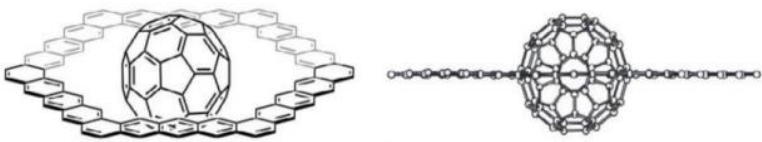

Figure 6 Supramolecular structures of $\mathrm{C}_{60}$ and [24]circulene.

Toyota and co-workers reported a cyclo-2,7-anthrylene hexamer synthesized by the coupling reaction, and it can form a complex with $\mathrm{C}_{60}$ in solution and in the solid state as revealed by X-ray analysis and NMR spectroscopy. These results offer clear experimental evidence of the theoretical prediction of the 
presence of nano-Saturns with disk-type hydrocarbon rings. ${ }^{[18]}$

After that, they reported the macrocyclic compounds composed of six anthracene units formed saturn-like complexes with fullerene $\mathrm{C}_{70}$ as ellipsoidal objects, and found that the binding constant of host-guest interaction between them was twice that of $\mathrm{C}_{60}$. Despite the different relative orientations of the $\mathrm{C}_{70}$ in the macrorings, the host-guest interaction is still similar, revealing the great versatility of the host for non-spherical fullerenes. ${ }^{[19]}$

Moreover, the internal "spheres" can be divided into two categories, one is hollow fullerene, and another is metallofullerene. Hollow fullerenes are mainly $\mathrm{C}_{60}$ and $\mathrm{C}_{70}$, which have been widely studied in many host-guest systems. The endohedral metallofullerenes have metal cluster(s) embedded in fullerene cage, which greatly enrich the structures and properties of fullerene materials. ${ }^{[2]}$ When the endohedral metallofullerene and the carbon nanoring form a supramolecular complex, different host-guest interactions may occur.

In 2014, Nakanishi et al. demonstrated the selective complexation of $\mathrm{C}_{82}$-based metallofullerenes with [11]CPP. They found that [11]CPP has a high affinity for $\mathrm{C}_{82}$-based metallofullerenes. ${ }^{[20]}$ Furthermore, the complexation can be

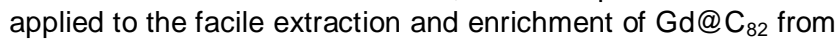
crude mixture. Later, Iwamoto et al. reported [11]CPP selectively encapsulates $L a @ C_{82}$ to form the shortest possible metallofullerene-carbon nanotube (CNT) peapod, $\mathrm{La} @ \mathrm{C}_{82} \subset[11] \mathrm{CPP}$, in solution and in the solid state. ${ }^{[21]}$

In 2015, Itami et al. reported the $\mathrm{Li}^{+} @ \mathrm{C}_{60} \subset[10] \mathrm{CPP} \cdot \mathrm{X}^{-}$. $X$-ray crystallography confirmed the molecular structure of $\mathrm{Li}^{+} @ \mathrm{C}_{60} \subset[10] \mathrm{CPP} \cdot \mathrm{X}^{-}$and uncovered the formation of an ionic crystal (Figure 7). The strong charge-transfer interaction between [10]CPP and $\mathrm{Li}^{+} @ \mathrm{C}_{60}$ was confirmed by electrochemical measurement and spectroscopic analyses, resulting in significant delocalization of the positive charge to the whole complex. ${ }^{[22]}$
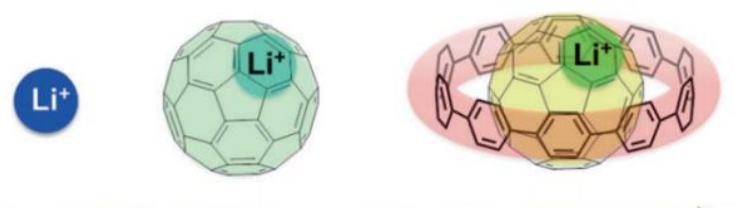

Localized

Positive charge

Delocalized

Figure 7 The change in positive-charge delocalization of $\mathrm{Li}^{+}$, $\mathrm{Li}^{+} @ \mathrm{C}_{60}$, and $\mathrm{Li}^{+} @ \mathrm{C}_{60} \subset[10] \mathrm{CPP}$ complex.

Interestingly, above systems exhibit varying degrees of charge-transfer behaviour. The use of [10]CPP as a supramolecular junction to generate modular dyads between a zinc porphyrin electron donor and five representative fullerene electron acceptor was recently described by Jasti's group with Guldi and Drewello. The syntheses of these systems are typically non-trivial, as they require a covalent linkage between the porphyrin and fullerene components. A porphyrinfunctionalized [10]CPP was used to to encapsulate $C_{60}$, allowing for through-space charge transfer between the porphyrin and fullerene (Figure 8). ${ }^{[23]}$

In 2020, Zhang et al. constructed supramolecular complexes of metallofullerenes and [12]CPP and investigated their properties. The selective encapsulation of three different sizes of metallofullerene $\mathrm{Sc}_{3} \mathrm{~N} @ \mathrm{C}_{78}, \mathrm{Sc}_{3} \mathrm{~N} @ \mathrm{C}_{80}$ and $\mathrm{Sc}_{2} \mathrm{C}_{2} @ \mathrm{C}_{82}$ by [12]CPP was disclosed. ${ }^{[24]}$ These studies provide a strategy to hoop the spherical metallofullerenes and modulate their electronic and magnetic properties using a circular nanoring. Metal-induced orientation and self-assembly characters for these supramolecular complexes reveal a great significance

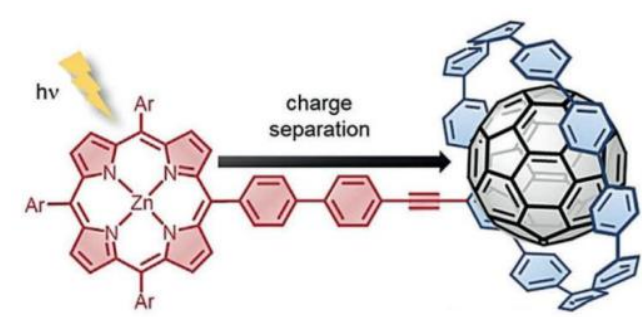

Figure 8 Supramolecular structure of porphyrin-functionalized [10]CPP and $\mathrm{C}_{60}$ with charge-transfer behaviour.

in molecule science and nanotechnology.

In 2019, Zhao et al. used the [12]CPP to hoop the $\mathrm{C}_{80}$-based metallofullerene $\mathrm{Y}_{3} \mathrm{~N} @ \mathrm{C}_{80}$ and azafullerene $\mathrm{Y}_{2} @ \mathrm{C}_{79} \mathrm{~N}$, resulting in a stable supramolecular complex, induced molecule orientation, changed assembly behavior, and tunable spin state. Moreover, the electron spin in paramagnetic $\mathrm{Y}_{2} @ \mathrm{C}_{79} \mathrm{~N}$ was employed to percept the host-guest interaction, and it showed anisotropic spin-metal couplings due to its insufficient rotational averaging in a confined space of the nanoring. The special spin character of $\mathrm{Y}_{2} @ \mathrm{C}_{79} \mathrm{~N} \subset[$ [12]CPP was also investigated in its solid state, and it exhibits the independent spin of $Y_{2} @ C_{79} N$ separated by a nanoring. ${ }^{[25]}$ Then they found that when the $\mathrm{Y}_{2} @ \mathrm{C}_{79} \mathrm{~N}$ and [4]CHBC are combined, a new peak will appear, revealing a strong host-guest interaction. ${ }^{[26]}$

\section{Conclusions and Perspectives}

Saturn-like supramolecular systems have been constructed by various carbon nanoring and spherical fullerene molecules through host-guest interactions. The carbon nanoring molecules are designed and synthesized by organic chemists. Fullerenes are selected to encapsulate into the cavity of nanoring to form varied saturn-like supramolecular complexes. The properties of host-guest interactions, charge-transfer behaviour, fluorescent emission, magnetic modulation, photoconductivity, etc., have been revealed in these Saturn-like supramolecular systems. Although the syntheses of new carbon nanorings and related supramolecular complexes have been developed rapidly in the past decade, the applications of these supramolecular complexes in the fields of electronic devices, molecular sensing and molecular machines still need to be further explored. For example, charge transfer is often accompanied by the formation of these supramolecular complexes, and it can result in high photoconductivity for their films. In addition, the unique magnetic sensing effect of paramagnetic metallofullerenes in Saturn-like supramolecular complexes can be used to design molecular magnetic probe as well as quantum bit. Moreover, it is reasonable to expect that these Saturn-like supramolecular complexes will attract huge research interests, and lead to many unprecedented applications as nanomaterials in the future.

\section{Conflict of Interest}

The authors declare no conflict of interest.

Copyright (C 2021 Jie Zhang, Chunru Wang, Taishan Wang. This article is an open access article distributed under the terms and conditions of the Creative Commons Attribution (CC BY) license (http://creativecommons.org/licenses/by/4.0/). The use, distribution or reproduction in other forums is permitted, provided the original author(s) or licensor are credited and that the original publication in this journal is cited, in accordance with accepted academic practice. No use, distribution or reproduction is permitted which does not comply with these terms. 


\section{References}

[1] Kroto, H. W.; Allaf, A. W.; Balm, S. P. C 60 : Buckminsterfullerene. Nature 1985, 91, 1213.

[2] Shinohara, H. Endohedral metallofullerenes. Rep. Prog. Phys. 2000, 63, 843.

[3] Sota, S.; Takashi, Y; Hiroyuki, I. Solid-state structures of peapod bearings composed of finite single-wall carbon nanotube and fullerene molecules. Proc. Natl. Acad. Sci. U. S. A. 2014, 111, 8374-8379.

[4] Wu, D.; Cheng, W.; Ban, X. T.; Xia, J. L. Cycloparaphenylenes (CPPs): An Overview of Synthesis, Properties, and Potential Applications. J. Org. Chem. 2018, 7, 2161-2181.

[5] Yuan, K.; Guo, Y. J.; Yang, T.; Dang, J. S.; Zhao, P; Li, Q. Z., Zhao, $X$. Theoretical insights into the host-guest interactions between [6]cycloparaphenyleneacetylene and its anthracene-containing derivative and fullerene $\mathrm{C}_{70}$. J. Phys. Org. Chem. 2014, 27, 772-782.

[6] Toyota, S.; Tsurumaki, E. Exploration of Nano-Saturns: A Spectacular Sphere-Ring Supramolecular System. Chem.-Eur. J. 2019, 25, 6878-6890.

[7] Evans; Paul, J.; Jasti, R. Molecular Belts. Top. Curr. Chem. 2014, 349, 249-290.

[8] Kawase, T.; Tanaka, K.; Seirai, Y.; Shiono, N.; Oda, M. Complexation of Carbon Nanorings with Fullerenes: Supramolecular Dynamics and Structural Tuning for a Fullerene Sensor. Angew. Chem. Int. Ed. 2003, 115, 5755-5758.

[9] Xu, Y. Z.; Delius, M. V. The Supramolecular Chemistry of Strained Carbon Nanohoops. Angew. Chem. Int. Ed. 2020, 59, 559-573.

[10] Jasti, R.; Bhattacharjee, J.; Neaton, J. B.; Bertozzi, C. R. Synthesis, Characterization, and Theory of [9]-, [12]-, and [18]Cycloparaphenylene: Carbon Nanohoop Structures. J. Am. Chem. Soc. 2008, 130, 17646-17647.

[11] Iwamoto, T.; Watanabe, Y.; Sadahiro, T.; Haino, T.; Yamago, S. Size-selective encapsulation of $\mathrm{C}_{60}$ by [10]cycloparaphenylene: formation of the shortest fullerene-peapod. Angew. Chem. Int. Ed. 2011, 123, 8492-8494.

[12] Yuan, K.; Guo, Y. J.; Zhao, X.; Nature of Noncovalent Interactions in the $[n]$ Cycloparaphenylene $\supset$ C70 $(n=10,11$, and 12) Host-Guest Complexes: A Theoretical Insight into the Shortest $\mathrm{C}_{70}-$ Carbon Nanotube Peapod. J. Phys. Chem. C. 2015, 119, 5168-5179.

[13] Xu, Y. Z.; Kaur, R.; Wang, B. Z.; Minameyer, M. B.; Gsanger, S.; Meyer, B.; Drewwllo. T.; Guldi, D. M.; Delius, M. V. ConcaveConvex pi-pi Template Approach Enables the Synthesis of [10]Cycloparaphenylene-Fullerene [2]Rotaxanes. J. Am. Chem. Soc. 2018, 140, 13413-13420.

[14] Rio, J.; Beeck, B.; Rotas, G.; Ahles, S.; Jacquemin, D.; Tagmatarchis, N.; Ewels, C.; Wegner, H. A. Electronic Communication between two [10]cycloparaphenylenes and
Bis(azafullerene) $\left(\mathrm{C}_{59} \mathrm{~N}\right)_{2}$ Induced by Cooperative Complexation. Angew. Chem. Int. Ed. 2018, 57, 6930-6934.

[15] Huang, Q.; Zhuang, G. L; Jia, H. X.; Qian, M. M.; Cui, S. S.; Yang, S. F.; Du, P. W. Photoconductive Curved-Nanographene/Fullerene Supramolecular Heterojunctions. Angew. Chem. Int. Ed. 2019, 58, 6244-6249.

[16] lyoda, M.; Shimizu, H. Multifunctional m-expanded oligothiophene macrocycles. Chem. Soc. Rev. 2015, 44, 6411-6424.

[17] Kigure, S.; Haruka, Q.; Hisanori, S.; Susumu, O. Nano-Saturn: Energetics of the Inclusion Process of $\mathrm{C}_{60}$ into Cyclohexabiphenylene. J. Phys. Chem. C. 2015, 119, 8931-8936.

[18] Yamamoto, Y.; Tsurumaki, E.; Wakamatsu, K.; Toyota, S. Nano-Saturn: Experimental Evidence ofComplex Formation of an Anthracene Cyclic Ring with C60. Angew. Chem. Int. Ed. 2018, 57, 8199-8202.

[19] Toyota, S.; Yamamoto, Y.; Wakamatsu, K.; Tsurumaki, E.; Muñoz Castro, A. Nano-Saturn with an Ellipsoidal Body: Anthracene Macrocyclic Ring-C ${ }_{70}$ Complex. Bull. Chem. Soc. Jpn. 2019, 92 1721-1728.

[20] Nakanishi, Y.; Omachi, H., Matsuura, S.; Miyata, Y.; Kitaura, R.; Segawa, Y.; Itami, K.; Shinohara, H. Size-Selective Complexation and Extraction of Endohedral Metallofullerenes with Cycloparaphenylene. Angew. Chem. Int. Ed. 2014, 53, 3102-3106.

[21] Iwamoto, T.; Slanina, Z.; Mizorogi, N.; Guo, J. D.; Akasaka, T.; Nagase, S.; Takaya, H.; Yasuda, N.; Kato, T.; Yamago, S. Partial Charge Transfer in the Shortest Possible Metallofullerene Peapod, La@C82ᄃ[11]Cycloparaphenylene. Chem.-Eur. J. 2014, 20, 1-8.

[22] Ueno, H.; Nishihara, T.; Segawa, Y.; Itami, K. Cycloparaphenylenebased ionic donor-acceptor supramolecule: isolation and characterization of $\mathrm{Li}(+) @ \mathrm{C}_{60}$ subset[10]CPP. Angew. Chem. Int Ed. 2015, 54, 3707-3711.

[23] Leonhardt, E. J.; Jasti, R. Emerging applications of carbon nanohoops. Nat. Rev. Chem. 2019, 3, 672-686.

[24] Zhang, J.; Zhao, C.; Meng, H. B.; Nie, M. Z.; Li, Q.; Xiang, J. F.; Zhang, Z. X.; Wang, C. R.; Wang, T. S. Size-selective encapsulation of metallofullerenes by [12]Cycloparaphenylene and dissociation using metal-organic framework. Carbon 2020, 161, 694-701.

[25] Zhao, C.; Meng, H. B.; Nie, M. Z.; Wang, X.; Cai, Z. F.; Chen, T.; Wang, D.; Wang, C. R.; Wang, T. S. Supramolecular Complexes of $\mathrm{C}_{80}$-Based Metallofullerenes with [12]Cycloparaphenylene Nanoring and Altered Property in a Confined Space. J. Phys. Chem. C 2019, 123, 12514-12520.

[26] Zhao, C.; Meng, H.; Nie, M.; Huang, Q.; Du, P.; Wang, C.; Wang, T. Chem. Commun. 2019, 55, 11511-11514.

Received October 13, 2021 Accepted December 9, 2021 